\title{
Merging Photonic Metamaterial and Optical Fiber Technologies
}

\author{
Angelos Xomalis ${ }^{1}$, Davide Piccinotti ${ }^{1}$, Artemios Karvounis ${ }^{1}$, I. Demirtzioglou ${ }^{1}$, Vassili Savinov ${ }^{1}$, Behrad \\ Gholipour $^{1,2}$, Jun-Yu Ou ${ }^{1}$, Yongmin Jung ${ }^{1}$, Eric Plum ${ }^{1}$, P. Petropoulos ${ }^{1}$, Kevin F. MacDonald ${ }^{1}$, David J. \\ Richardson', Nikolay I. Zheludev ${ }^{1,3}$ \\ 1. Optoelectronics Research Centre and Centre for Photonic Metamaterials, University of Southampton, SO17 1BJ, UK \\ 2. Chemistry, University of Southampton, SO17 1BJ, UK \\ 3. Centre for Disruptive Photonic Technologies, School of Physical and Mathematical Sciences \& The Photonics Institute, \\ Nanyang Technological University, Singapore 637371, Singapore \\ kfm@orc.soton.ac.uk; www.nanophotonics.org/niz
}

\begin{abstract}
We report on approaches to the integration of metasurfaces and active metadevices with optical fiber platforms, including all-optical and electro-optic nano-mechanical switching devices, dispersion control solutions and coherent absorption modulators.

OCIS codes: (160.3918) Metamaterials; (060.2310) Fiber optics; (230.3120) Integrated optics devices
\end{abstract}

In recent years, the field of photonic metamaterials research has migrated from the study of almost exclusively plasmonic metal nanostructures to embrace a variety of advanced material platforms, including semiconductors, superconductors, transparent conductive oxides, nitrides, phase-change media, and topological insulators. Opticallyand electronically-actuated reconfigurable photonic metasurfaces based on such materials offer a range of low-loss, nonlinear, tunable and switchable optical functionalities in ultra-compact form-factor, greatly extending the already remarkable range of unusual and enhanced optical properties available 'on demand' [1] via the metamaterials paradigm - for example, engaging nano(opto)mechanical [2], phase-change [3], and coherent control [4] response mechanisms to serve signal modulation, spectral/polarization selection or dispersion manipulation applications.

Realizing their full applications potential though, requires in many cases the integration of metasurface structures and metadevices with existing photonic and optoelectronic technology platforms, not least optical fibers - whereby they may serve a variety of ICT and sensing applications. Here we review recent advances in metamaterials research towards this goal, considering several approaches to the integration of functional nanostructures with optical fibers.

'Single-ended' fiber-optic metadevices, for example, may be realized by fabricating metasurface nanostructures directly on the end facets of cleaved fibers, as illustrated in Fig. 1, which shows a dispersion-control silicon metasurface manufactured on a single-mode fiber tip, with sharply resonant transmission properties set by structural design.
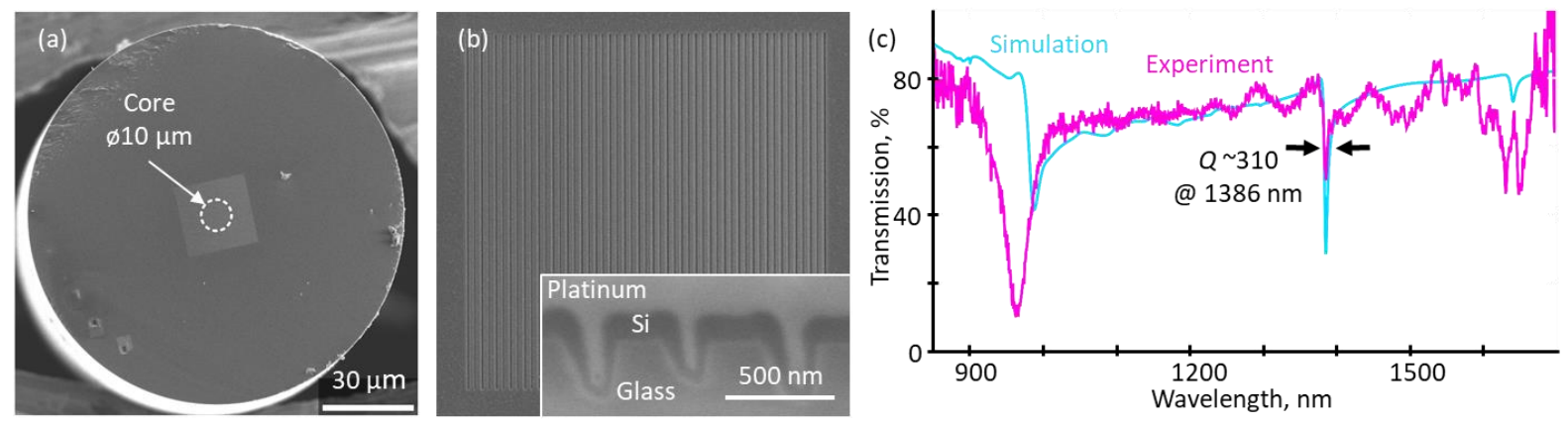

Fig. 1. Si metasurface on polarization-maintaining single-mode fiber. (a) Scanning electron microscope image of a fiber end facet upon which a silicon metasurface [sub-wavelength-period binary grating] has been manufactured over the core area. The metasurface structure is defined in the glass of the fiber by focused ion beam milling and then coated with a thin film of Si by chemical vapor deposition. (b) Closer detail of the metasurface [period $930 \mathrm{~nm}$ ], with cross-sectional image inset [Pt being deposited over the Si only for the purposes of sectioning]. (c) Experimentally measured and numerically modelled spectral dispersion of metasurface transmission. 
Micro-collimator assemblies may be employed for the realization of 'in-line' metadevices wherein planar, e.g. free-standing nano-mechanically reconfigurable membrane, metasurfaces are coupled to a pair of fibers - one on either side. This in-line integration approach has been engaged for the realization of a high-bandwidth, fiberized alloptical gate exploiting coherent control of absorption in a plasmonic thin film (70 nm gold) metamaterial (Fig. 3). With appropriate settings of the intensity and relative phase of the two input signals, the device can be configured to perform light-by-light control operations analogous to those of electronic AND, NOT and XOR gates. This coherent control functionality is based solely on principles of linear interference (i.e. requires no intrinsically nonlinear media) and offers modulation bandwidth up to $100 \mathrm{THz}$ [5] at arbitrarily low (even single-photon [6]) energy levels.
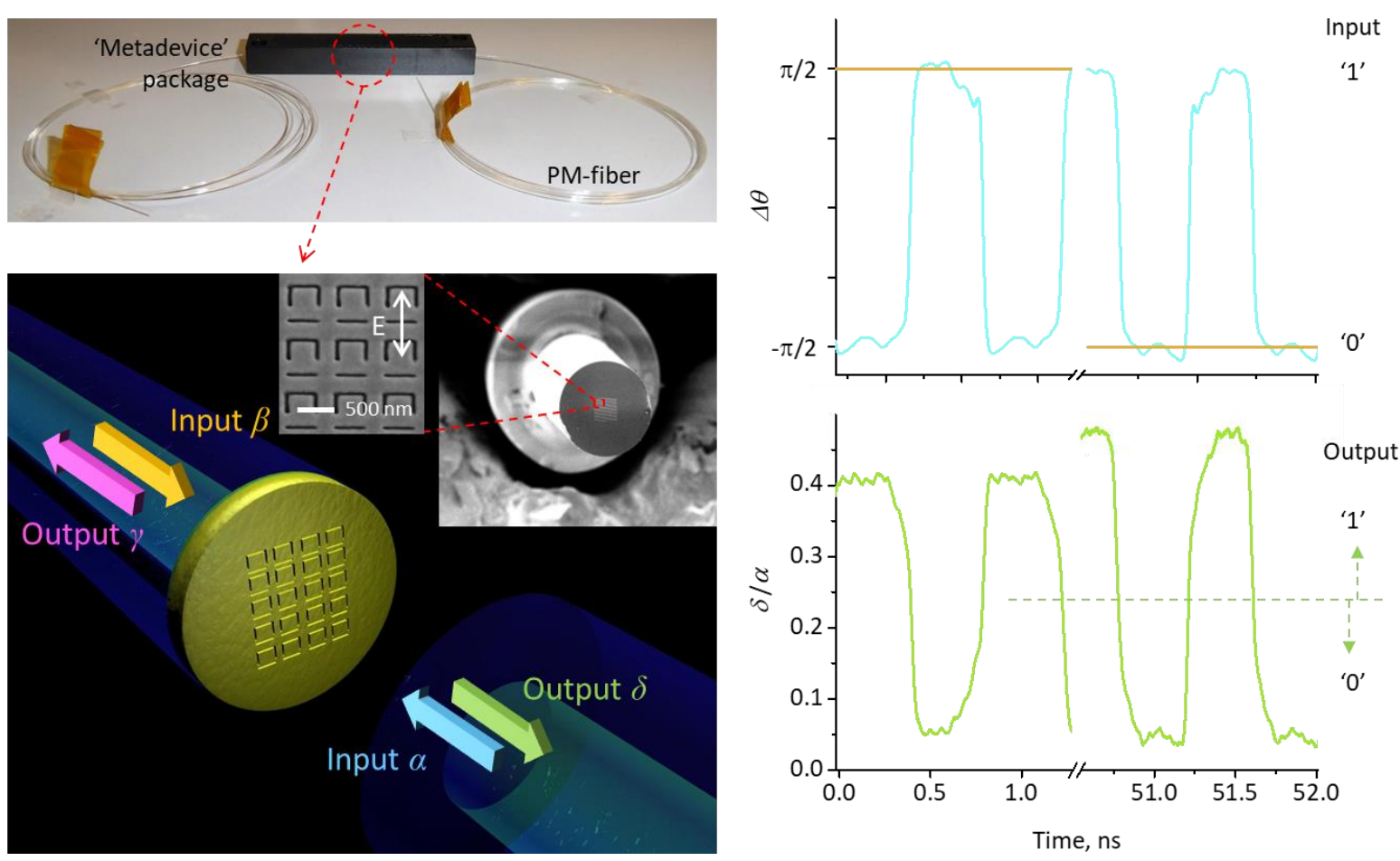

Fig. 2. Fiberized coherent absorption modulator. Operational schematic [lower left, excluding micro-lens collimator elements for clarity] of the 4-port device configuration comprising two counter-propagating input incident on a sub-wavelength thickness metamaterial absorber fabricated on the end facet of a polarization-maintaining fiber [inset scanning electron microscope images], and two corresponding output beams. Input signals $\alpha$ and $\beta$ [upper right] - logical levels ' 0 ' and ' 1 ' encoded in relative phase at the metamaterial plane, and corresponding output signal $\delta$ [lower right; amplitude relative to $\alpha$ ] for such a device configured to perform an XOR operation, i.e. to give an output ' 1 ' only when the two inputs are different.

\section{References}

[1] N. I. Zheludev, "Obtaining optical properties on demand," Science 348, 973-974 (2015).

[2] N. I. Zheludev and E. Plum, "Reconfigurable nanomechanical photonic metamaterials," Nat. Nanotech. 11, 16-22 (2016).

[3] A. Karvounis, B. Gholipour, K. F. MacDonald and N. I. Zheludev, "All-dielectric phase-change reconfigurable metasurface," Appl. Phys. Lett. 109, 051103 (2016).

[4] X. Fang, K. F. MacDonald and N. I. Zheludev, "Controlling light with light using coherent metadevices: all-optical transistor, summator and invertor," Light Sci. Appl. 4, e292 (2015).

[5] V. Nalla, S. Vezzoli, J. Valente, H. Sun and N. I. Zheludev, "100 THz optical switching with plasmonic metamaterial," in CLEO/Europe-EQEC 2015 (Munich, Germany, 2015)

[6] T. Roger, S. Vezzoli, E. Bolduc, J. Valente, J. J. F. Heitz, J. Jeffers, C. Soci, J. Leach, C. Couteau, N. I. Zheludev and D. Faccio, "Coherent perfect absorption in deeply subwavelength films in the single-photon regime," Nat. Commun. 6, 7031 (2015). 